\title{
Studies on Rheological Behavior of Xanthan Gum Solutions in Presence of Additives
}

Khan SY1, Yusuf $\mathbf{M}^{2 *}$ and Sardar $\mathrm{N}^{1}$

1Department of Petroleum Studies, ZH College of Engineering and Technology, AMU, Aligarh, India

2Department of Petroleum Engineering, The Glocal University, Saharanpur, India

\section{Research Article}

Volume 2 Issue 5

Received Date: July 19, 2018

Published Date: August 13, 2018

*Corresponding author: Mohd Yusuf, Department of Petroleum Engineering, The Glocal University, Saharanpur, India, Email: yusuf@theglocaluniversity.in

\section{Abstract}

The oil and gas sector is one of the six core industries in India. These industries are of strategic importance and plays a vital role in influencing decisions across other important spheres of the economy. But the overall production has declined due to the increase in maturity of the oil reservoirs. In developing countries like India, the oil production and demands play a crucial role for the development of economy of the country. However the domestic crude oil production is insufficient to meet the requirement of energy. Thus, there is the big challenge to minimize the gap between demand and supply of crude oil. Several methods to enhanced oil recovery have been developed to increase the production from matured reservoirs and are referred to enhance oil recovery (EOR) methods. In the present study we have studied the rheological behaviour of Xanthan gum solution in absence and presence of surfactants and sacrificial agents such as salt and alkali. Xanthan gum is a polysaccharide and form an aqueous solution with high viscosity and show non-Newtonian behaviour and can be used in oil production as drilling fluids and in enhance oil recovery. The power law model was used to describe the pseudo-plastic behaviour of polyacrylamide solution. The additives shows synergistic effects and their concentration strongly influence the shear viscosity of the solution. The TEM results support the viscosity data. The results obtained are quite interesting and could be of prolific use for selection of polymer-surfactant mixtures and surfactants for use in EOR.

Keywords: Xanthan Gum; Crude oil; Fluids; Reservoirs; Viscosity

Abbreviations: EOR: Enhance Oil Recovery; SDS: Sodium Dodecyl Sulfate; TEM: Transmission Electron Microscopy.

\section{Introduction}

Oil, natural gas and coal are the main constituents of fossil fuel energy. These energy resources were formed millions of years ago due to the decomposition of organic 


\section{Petroleum \& Petrochemical Engineering Journal}

matters which remains trapped in the sediments and subjected to the conditions of high temperature and pressure under the earth crust. Hypothetically, estimated place crude oil of the world are of the order of 6,000 billon barrel (B. barrel $\sim 159$ L) which includes the recoverable and non-recoverable oil in the reservoirs, of which about 3,000 BB could be technically recovered and produced [1]. Further, we all know that about two-third of the oil remains in reservoir on an average after the natural drive and secondary recovery methods. This residual oil is trapped in the porous structure of reservoirs by capillary forces which cannot be recovered by conventional methods. Due to the increasing demand of energy and diminishing amount of conventional oil, companies have been relying on enhanced oil recovery methods for decades. Presently, lots of researches are being carried out globally to improve enhanced oil recovery techniques to fulfil demands of oil. Enhanced oil recovery methods, also known as tertiary oil recovery techniques and are mainly of the following categories: Thermal methods, Gas methods and Chemical EOR methods.

From literature review, we found that in EOR by using certain combinations of different surfactants and flow modify water soluble polymers, higher displaced efficiencies can be obtained than by the individual component alone. Further, the varying condition of salinity and alkalinity in oil well is one of the reasons to study the effect of additives on the rheology of polymersurfactant solutions. The effect of such condition on polymer solution is important because they can alter its rheological properties. Therefore in the present study, solution behaviour of xanthan gum, a viscosity enhancer polymer in presence of an anionic surfactant Sodium Dodecyl Sulfate (SDS) was studied by rheometry with special interest of EOR. Polymers are often used which are often used as mobility controller for EOR. Injection of small quantity of polymer significantly increases the viscosity of solution, which can increase the sweep efficiency of the displacing fluid in the porous media during flooding. The aqueous solution of polymer shows non- Newtonian behaviour and its apparent viscosity is function of polymer concentration, shear rate, temperature, etc [2].

\section{Materials and Methods}

The polymer, surfactant and other chemicals used in the present investigation are:

Xanthan Gum, a biopolymer. Sodium dodecyl sulfate (SDS), an anionic surfactant, Anton Paar (Rheometer).

\section{Experimental Methods}

The techniques used in the present study are:

1. Rheometry: The rheological parameters were measured with the help of a modular compact rheometer (MCR 102 Anton Paar Germany) equipped with a temperature control system with an accuracy of $0.01^{\circ} \mathrm{C}$. The techniques involve the application of a known strain or strain rate to a sample and the subsequent measurement of the stress induced in the sample or vice versa. The viscosity curve can be obtained with varying share rate from $0.01 \mathrm{~s}^{-1}$ to $1000 \mathrm{~s}^{-}$ 1 .

2. Transmission Electron Microscopy (TEM): Transmission electron microscopy (TEM) is a microscopy technique in which a beam of electrons is transmitted through an ultra-thin specimen, interacting with the specimen as it passes through.

\section{Results and Discussion}

The results of present study can be discussed as:

1. Effects of Xanthan gum concentration on viscosity of its aqueous solution.

2. Validation of the Power-law model.

3. Effects of Sodium dodecyl sulfate (SDS) concentration on viscosity of Xanthan gum's aqueous solution.

4. Effects of temperature on viscosity of Xanthan gum solutions at different concentration.

5. Transmission Electron Microscopy (TEM) Analysis for Surface Morphology

\section{Rheometry Studies}

Viscosity profiles for non-Newtonian shear-dependent behaviour of Xanthan gum solutions were measured in order to study the solution behaviour of Xanthan gum in the absence and presence of surfactant, salts and alkali. The results of present study are presented and discussed as:

\section{Rheometry Results For Pure Xanthan Gum Solutions}

\section{Effect of Concentration of Xanthan Gum on Viscosity}

The flow curves for aqueous solution of Xanthan gum at different temperature and varying shear rate $\left(0-1000 \mathrm{~s}^{-}\right.$ 1) are shown in Figure 1. An increase in shear viscosity was observed for the increased xanthan gum concentration. For polymer flooding in the EOR, the increased shear viscosity of the xanthan gum can increase the residual resistance factor and improve the sweep efficiency as reported earlier [3]. The viscosity of all 


\section{Petroleum \& Petrochemical Engineering Journal}

samples decreases with increasing shear rate showing the shear thinning behaviour of xanthan gum solution at all concentrations. From figure 1 it can be observed that at all concentrations $(0.10 w \mathrm{t} \%, 0.25 \mathrm{wt} \%, 0.50 \mathrm{wt} \%)$ and all temperatures $(303 \mathrm{~K}, 313 \mathrm{~K}, 323 \mathrm{~K})$ the aqueous solution of pure Xanthan gum shows similar flows pattern. Significant shear-thinning behaviour was observed, which was attributed to the dis-entanglement and alignment of the xanthan gum polymer chains along the flow direction. In a drilling fluid of the EOR, shear thinning polymer solutions were designed that can suspend drilling cutting at low shear rates, but offer little resistance to flow at high shear rates. On the other hand, polysaccharides that display interesting solution characteristics tend to form ordered structures in aqueous environments. Large molecules in an aqueous solutions form aggregates and entangle, leading to high viscosity at low shear rates. The increase of viscosity with increasing xanthan gum concentration has been attributed to intermolecular interaction or entanglement that increases the effective macromolecule dimensions and molecular weight [4]. Increasing the shear rate decreases the steady shear viscosity due to the uncoiling and partial alignment of the polymer chains at the high shear rate region. Xanthan gum solutions are highly pseudo plastic. Although they show a shear-thinning behaviour under an applied shear rate, the initial viscosity is recovered almost instantaneously when the shear rate is removed [5].
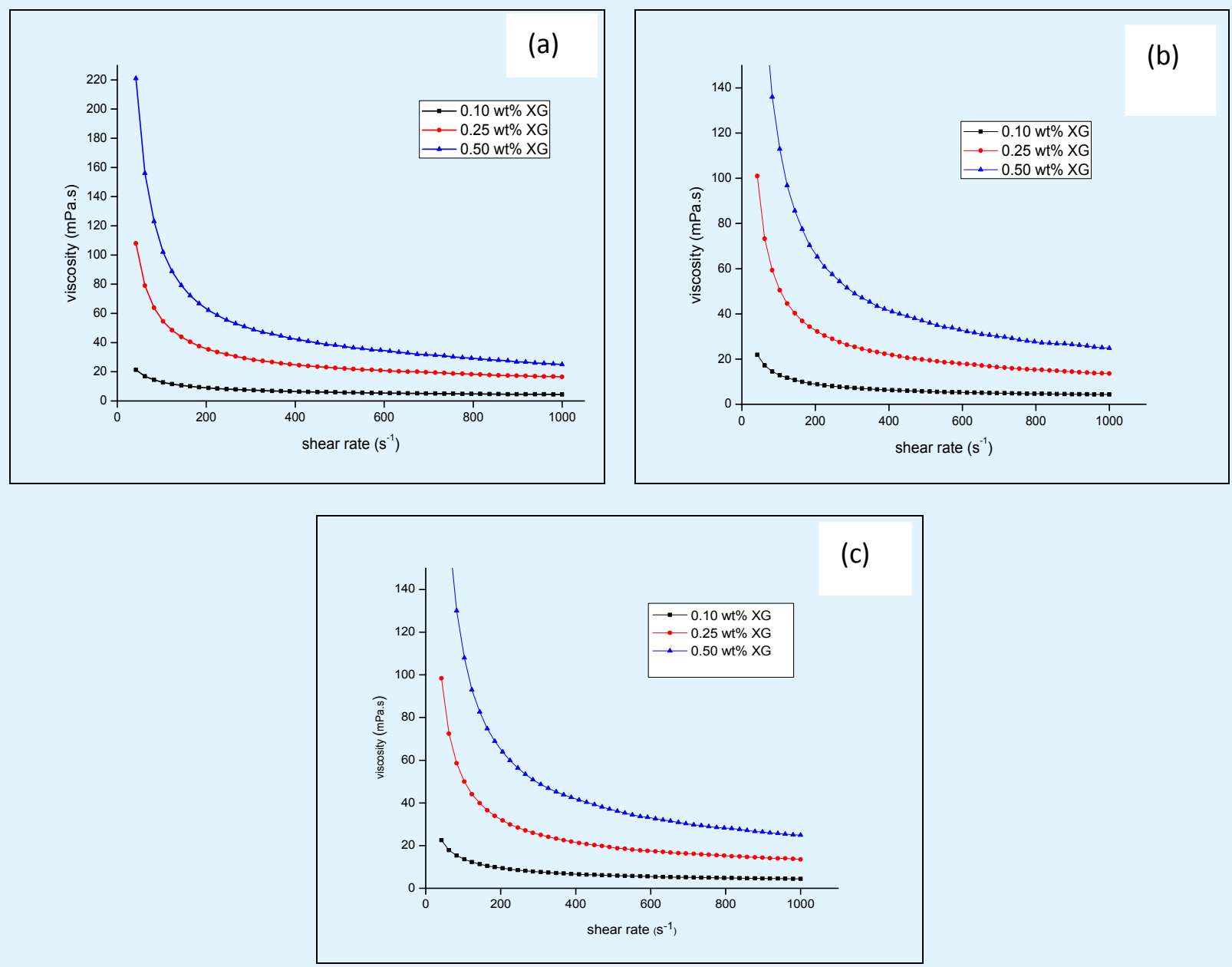

Figure 1: Plot of Viscosity vs shear rate for different Xanthan Gum concentrations (a) at $303 \mathrm{~K}$ (b) at $313 \mathrm{~K}$ (c) at $323 \mathrm{~K}$. 


\section{Petroleum \& Petrochemical Engineering Journal}

The shear thinning behaviour of xanthan gum in deionized water solutions is shown in figure1. The viscosity at high shear rate $\left(1000 \mathrm{~s}^{-1}\right)$ was 3.30 times less than the magnitude of viscosity at low shear rate $\left(100 \mathrm{~s}^{-1}\right)$ for $0.25 \mathrm{wt} \%$ Xanthan gum at $303 \mathrm{~K}$. Figure 2 shows the plot of viscosity as a function of Xanthan gum concentration at an arbitrary shear rate $\left(160 \mathrm{~s}^{-1}\right)$ where the curves were found to fit the power law model well. We can see from graphs that the viscosity at low shear rate increased linearly with increasing xanthan gum concentration within the range of $0.01 \mathrm{wt} \%$ to $0.5 \mathrm{wt} \%$ as explained by earlier investigators [5]. The static viscosity and shear thinning behavior of xanthan gum solution are related but not proportional to xanthan gum concentration over a wide range. Choppe et al. reported an exponential relationship of the solution viscosity with xanthan gum concentration when it was higher than $2000 \mathrm{mg} / \mathrm{L}[6]$.

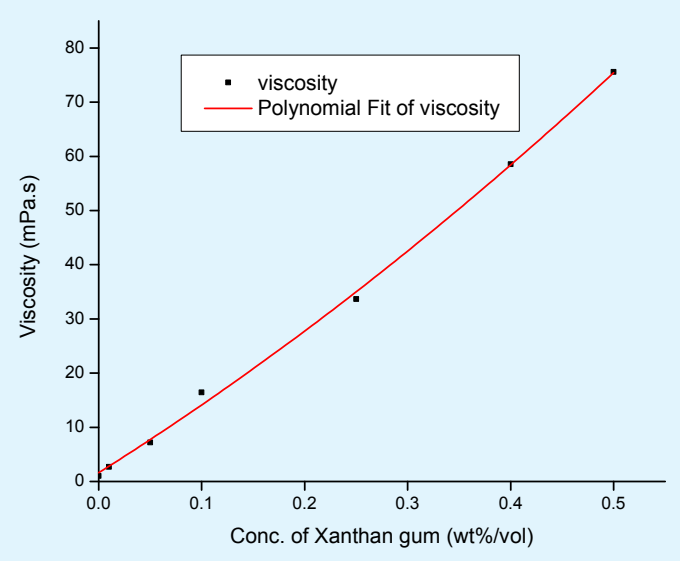

Figure 2: Plot of Viscosity vs Xanthan gum concentration at $160 \mathrm{~s}^{-1}$ and $303 \mathrm{~K}$.

\section{Validity of Power-Law Model}

Xanthan gum solutions are classified under pseudoplastic fluid which means that resistance to flow decreases as the shear rate increases. The pseudoplastic behaviour of xanthan gum solution was examined and the power-law model (Equation 1) is used to describe this behaviour.

$$
\tau=K \gamma^{n}
$$

Where, $\tau$ is shear stress $(\mathrm{Pa}), \gamma$ is the shear rate $\left(\mathrm{s}^{-1}\right), \mathrm{n}$ is the flow behaviour index, and $\mathrm{K}$ is the consistency index $\left(\mathrm{Pa} . \mathrm{S}^{\mathrm{n}}\right)$. In this study value of shear stress was measured at varying shear rate and with the help of regression analysis and it was found that the value of $K$ and $n$ is 3.086 and 0.489 respectively for $0.25 \mathrm{wt} \%$ aqueous xanthan gum solution at $30^{\circ} \mathrm{C}$. It is well known that for pseudoplastic fluids, $n<1.0$. It should be noted that for Newtonian fluids the value of $n$ is unity and $\mathrm{K}$ became the dynamic viscosity, $\eta$. The fitted $K$ and $n$ value, as well as the coefficient of determination, $\mathrm{R}^{2}$ are listed in Table 1.

\begin{tabular}{|c|c|c|c|c|}
\hline $\begin{array}{c}\text { Xanthan gum } \\
\text { Conc. }\end{array}$ & $\mathbf{K}$ & $\mathbf{n}$ & $\mathbf{R}^{2}$ & ABS Deviation \\
\hline $0.25 \mathrm{wt} \% / \mathrm{vol}$. & 3.086 & 0.489 & 0.9919 & 0.02280 \\
\hline
\end{tabular}

Table 1: Fitted $K$ and $n$ values according to Equation (1) including $\mathrm{R}^{2}$ and $\mathrm{ABS}$ deviation value.

\section{Effects of Sodium Dodecyl Sulfate (SDS) Concentration on Viscosity of Aqueous Solution of Xanthan Gum}

Surfactants slugs are generally used in EOR processes to mobilize residual oil by changing the rock wettability and/or reducing the interfacial tension. To increase the efficiency of such process, polymers can be either coinjected with the surfactant slug or injected after it and there are chances of mixing of surfactants and polymers. Therefore, the effects of anionic surfactant (Sodium dodecyl sulfate, SDS) on viscosity of Xanthan gum solutions were examined. Figure 3 shows the effect of SDS concentration on viscosity of $0.1 \mathrm{wt} \%$ Xanthan gum solution at 303K. It was found that on increasing SDS concentration from 0 to $5 \mathrm{mM}$, a significant decrease in viscosity of Xanthan gum solution was observed. The apparent viscosity of polymer decreases with an increase in the surfactant concentration. These results indicate that SDS reacts physically as well as chemically with the polymer chain in deionized water. This trend is similar to that observed by Shupe [7] and suggests that the anionic surfactant affects the viscosity behaviour of the Xanthan gum through the charge-shielding mechanism but further addition of SDS had little effect on viscosity. Figure 4 shows the effect of SDS concentration on viscosity of $0.25 \mathrm{wt} \%$ Xanthan gum solution at fixed shear rate $\left(160 \mathrm{~s}^{-}\right.$ 1) where the curves were found to fit the power law model for 313K. We can also observed that the viscosity of solution first decreases on addition of SDS (5mM SDS) and further it became almost constant with very little effect on viscosity of solution. However, for $20 \mathrm{mM}$ SDS the viscosity of solution again decreases at low shear rate, but the behaviour is quite different at higher shear rate. 


\section{Petroleum \& Petrochemical Engineering Journal}

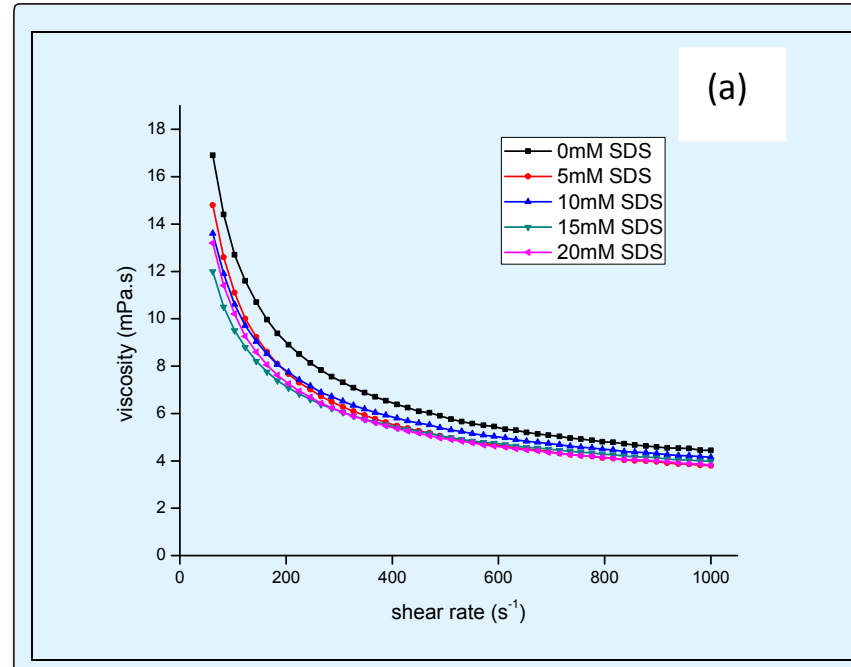

Figure 3: Viscosity vs shear rate of solution containing 0.1 wt \% Xanthan Gum and different SDS concentration at $303 \mathrm{~K}$.

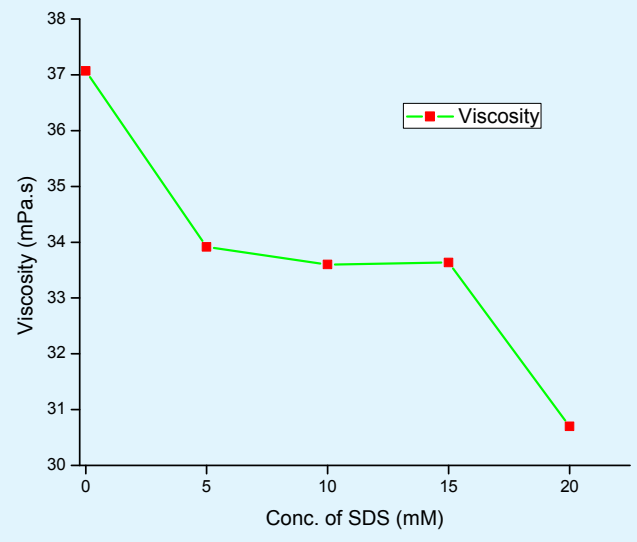

Figure 4: Effect of SDS concentration on viscosity of $0.25 \%$ Xanthan gum solution at fixed shear rate $\left(160 \mathrm{~s}^{-}\right.$ ${ }^{1}$ ) and at $313 \mathrm{~K}$.

\section{Effect of Temperature on Viscosity of Xanthan Gum Solution}

At all polymer concentrations examined, the solution viscosity diminished with temperature.

As the temperature increases, the average speed of molecules in liquid increases, so the time they spend in contact with their nearest neighbour decreases. Thus, as temperature increases, the average intermolecular forces decreases. The effect of temperature on Xanthan Gum viscosity is very complicated. As the solution temperature increases, the solvent viscosity decreases, but the degree of hydrolysis of xanthan gum increases. As the degree of hydrolysis increases, the viscosity of xanthan gum in deionized water increases as a result of the electrostatic repulsion between the negative charges on the polymer chain [8]. This effect of temperature on viscosity of Xanthan gum solution can be seen in Figure 5 \& 6 .

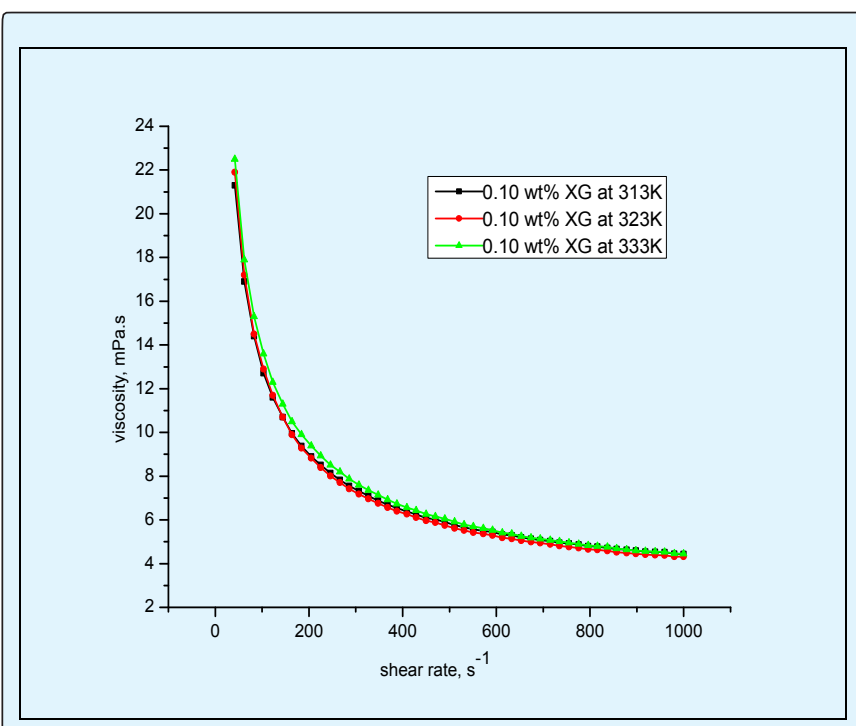

Figure5: Viscosity vs shear rate for $0.1 \mathrm{wt} \% \mathrm{XG}$ solution at $303 \mathrm{~K}, 313 \mathrm{~K}$ and $323 \mathrm{~K}$.

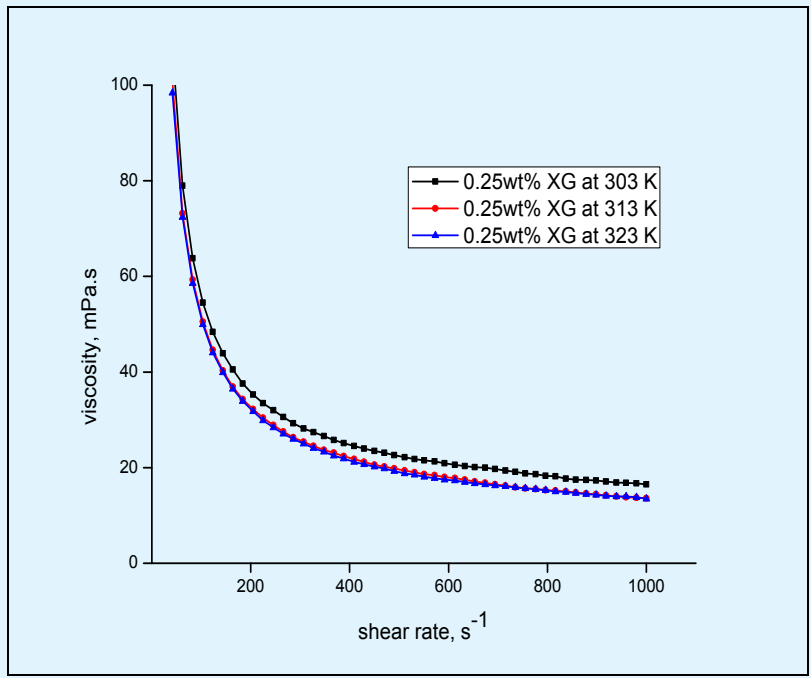

Figure 6: Viscosity vs shear rate for $0.25 \mathrm{wt} \% \mathrm{XG}$ solution at $303 \mathrm{~K}, 313 \mathrm{~K}$ and $323 \mathrm{~K}$. 


\section{Petroleum \& Petrochemical Engineering Journal}

The change in temperature for $0.10 \mathrm{wt} \%$ Xanthan gum solution do not shows significant effect on viscosity of solution, where as for $0.25 \mathrm{wt} \%$ and $0.5 \mathrm{wt} \%$ Xanthan gum increase in temperature shows significant decrease in viscosity of solution. It can be seen from figure 6 that for $0.25 w t \%$ Xanthan gum solution first viscosity decreases when temperature increases from $303 \mathrm{~K}$ to $313 \mathrm{~K}$, and for $323 \mathrm{~K}$ there is very small change in viscosity of gum solution. Whereas for $0.05 \mathrm{wt} \%$ Xanthan gum solution the flow curves are quite close at temperature $303 \mathrm{~K}$ and $313 \mathrm{~K}$ but when temperature increases to $323 \mathrm{~K}$, a significant decrease in viscosity of xanthan gum solution is observed. The figures shown below have viscosity vs shear rate plot in which viscosity is in units of mPa.s and shear rate is in $\mathrm{s}^{-1}$.

\section{Transmission Electron Microscopy (TEM) Analysis for Surface Morphology}

Figure 7 demonstrates typical TEM images of the Xanthan gum solution in presence of additives. Figure 7(a) shows the surface morphology for pure SDS system $(15 \mathrm{mM})$ here we can see micelles in the system, also pure SDS image appeared as smooth surface. Figure 7 (b) shows the surface morphology for $0.25 \mathrm{wt} \%$ Xanthan gum in presence of $15 \mathrm{mM}$ SDS. We can see that SDS is homogeneously dispersed into polymer forming polymersurfactant aggregates. Figure 7(c) presents TEM results for $0.25 w t \%$ Xanthan gum $+15 \mathrm{mM}$ SDS $+1 w t \% \mathrm{NaCl}$ system, here micelles move apart indicating charge shielding effects of $\mathrm{NaCl}$.

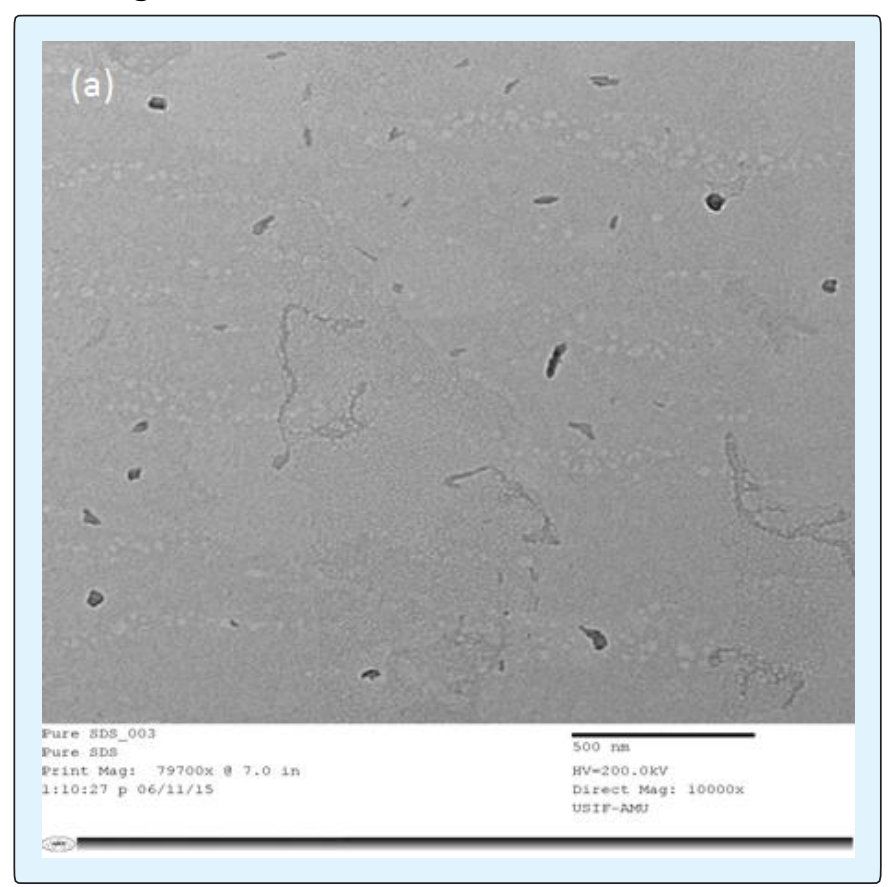

Yusuf M, et al. Studies on Rheological Behavior of Xanthan Gum Solutions in Presence of Additives. Pet Petro Chem Eng J 2018, 2(5): 000165
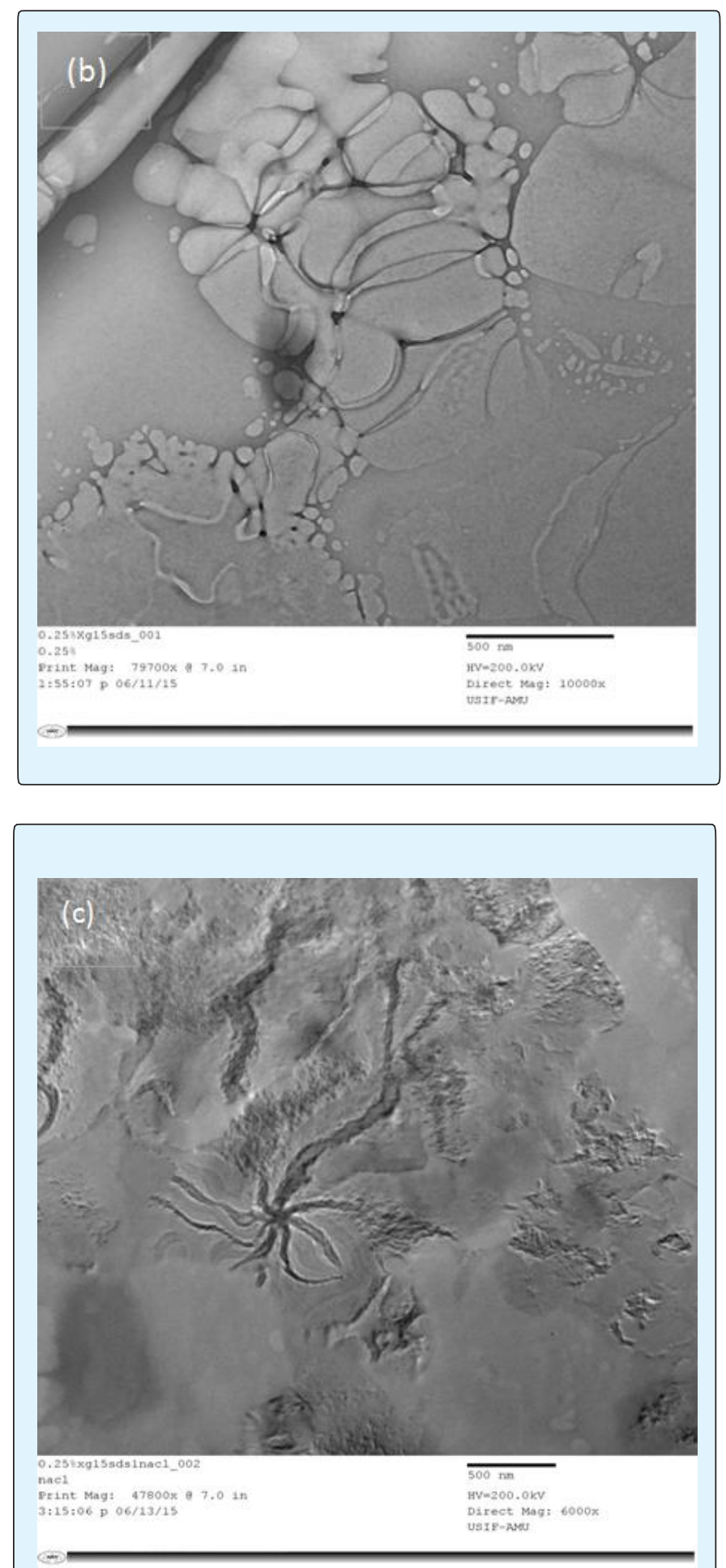

Figure 7: TEM Images of (a) Pure SDS (b) $0.25 w t \%$ Xanthan gum $+15 \mathrm{mM}$ SDS. (c) $0.25 w t \%$ Xanthan gum $+15 \mathrm{mM}$ SDS $+1 w \mathrm{t} \% \mathrm{NaCl}$ 


\section{Petroleum \& Petrochemical Engineering Journal}

\section{Conclusions}

Based on various experiments conducted during this research, following conclusions can be made:

1. Rheometry results for pure Xanthan gum shows that with increasing polymer concentrations the viscosity of solutions increases. At lower shear rate $\left(160 \mathrm{~s}^{-1}\right)$ an increase in five fold concentration of Xanthan gum (from 0.01 to $0.05 \mathrm{wt} \%$ ) gives $169 \%$ increase in viscosity at $303 \mathrm{~K}$. At higher concentrations of Xanthan gum (0.1 to $0.5 \mathrm{wt} \%)$ increase five fold in concentration, there is $127.7 \%$ increment in viscosity can be observed.

2. Increasing shear rate the viscosity of solution decreases which shows the shear thinning behaviour of Xanthan gum solutions at all concentration under study. Also it was observed that on $100 \%$ increase in low shear rate $\left(103 \mathrm{~s}^{-1}-206 \mathrm{~s}^{-1}\right)$, the viscosity of $0.25 \mathrm{wt} \%$ Xanthan gum solution at $303 \mathrm{~K}$ decreases by $35.22 \%$ but at higher shear rate $\left(450 \mathrm{~s}^{-1}-900 \mathrm{~s}^{-1}\right)$, decrease in viscosity is found to be $27.23 \%$.

3. The effect of temperature on Xanthan Gum viscosity was studied and found to be very complicated. As the solution temperature increases, the solvent viscosity decreases, but the degree of hydrolysis of xanthan gum increases. As the degree of hydrolysis increases, the viscosity of xanthan gum in deionized water increases. For lower concentration of Xanthan gum $(0.1 \mathrm{wt} \%)$ decrease in temperature does not shows significant decrease on viscosity of solution, whereas at higher concentration $0.25 \mathrm{wt} \%$ and $0.5 \mathrm{wt} \%$ of Xanthan gum solution increase in temperature show significant decrease in viscosity of solution.

4. Shear thinning behaviour of Xanthan gum is decreased with an increase in temperature.

5. The pseudoplastic behaviour of Xanthan gum solution is confirmed by Power Law model.

6. TEM result supports rheological studies and images also showed surfactant-polymer interaction.

\section{References}

1. Thakur NK, Rajput S (2011) Exploration of Gas Hydrates: Geophysical Techniques. 1 ${ }^{\text {st }}($ Edn. $)$, Springer-Verlag Berlin Heidelberg, pp: 281.

2. Nasr-El-Din HA, Hawkins BF, Green KA (1992) Recovery of Residual Oil Using the Alkali/Surfactant/Polymer Process: Effect of Alkali Concentration. J Pet Sci Eng 6(4): 381-401.

3. Yeon H, Zhang K, Hyun B, Jin H (2014) Enhanced Oil Recovery Performance and Viscosity Characteristics of Polysaccharide Xanthan Gum Solution. Journal of Industrial and Engineering Chemistry 21: 741-745.

4. Dintzis FR, Babcock GE, Tobin R (1970) Studies on Dilute Solutions and Dispersion of the Polysaccharide from Xanthomonas Campestris NRRL B-1459. Carbohydr Res 13(2): 257-267.

5. Zhong L, Oostrom M, Truex MJ, Vermeul VR, Szecsody JE (2013) Rheological Behavior of Xanthan Gum Solution Related to Shear Thinning Fluid Delivery For Subsurface Remediation. J Hazard Mater 244-245: 160-170.

6. Choppe E, Puaud F, Nicolai T, Benyahia L (2010) Rheology of Xanthan Solutions as a Function of Temperature, Concentration and Ionic Strength. Carbohydr Polym 82(4): 1228-1235.

7. Shupe R (1981) Chemical Stability of Polyacrylamide Polymers. J Pet Technol 33(8): 1513-1529.

8. ASP Flooding.

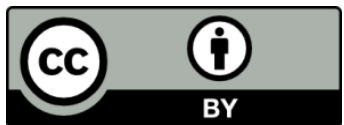

